\title{
Set-membership approach and Kalman observer based on zonotopes for discrete-time descriptor systems *
}

\author{
Ye Wang $^{\mathrm{a}}$, Vicenç Puig ${ }^{\mathrm{a}}$, Gabriela Cembrano ${ }^{\mathrm{a}, \mathrm{b}}$ \\ ${ }^{a}$ Advanced Control Systems (SAC) Research Group at Institut de Robòtica i Informàtica Industrial (IRI), CSIC-UPC, \\ Automatic Control Department, Universitat Politècnica de Catalunya-BarcelonaTech (UPC), \\ C/ Llorens i Artigas 4-6, 08028 Barcelona, Spain \\ ${ }^{\mathrm{b}}$ Cetaqua, Water Technology Centre, Ctra. d'Esplugues 75, Cornellà de Llobregat, 08940 Barcelona, Spain
}

\begin{abstract}
This paper proposes a set-membership state estimator and a zonotopic Kalman observer for discrete-time descriptor systems. Both approaches are developed in a set-based context considering system disturbances, measurement noise, and unknown inputs. This set-membership state estimation approach determines the set of consistent states with the model and measurements by constructing a parameterized intersection zonotope. Two methods to minimize the size of this intersection zonotope are provided: one inspired by Kalman filtering and the other based on solving an optimization problem involving a series of linear matrix inequalities. Additionally, we propose a zonotopic Kalman observer for discrete-time descriptor systems. Moreover, the relationship between both approaches is discussed. In particular, it is proved that the zonotopic Kalman observer in the current estimation type is equivalent to the set-membership approach. Finally, a numerical example is used to illustrate and compare the effectiveness of the proposed approaches.
\end{abstract}

Key words: Set-membership approach; Kalman observer; zonotopes; discrete-time descriptor systems; unknown inputs.

\section{Introduction}

In many industrial applications involving distribution or collection networks (as e.g. water and electrical networks), mass and energy balance static equations must hold. A standard model including only the dynamical part (described by ordinary differential/difference equations) is not enough to represent system dynamics subject to static relations among system variables. Such systems, known as descriptor systems (also known as singular, implicit or differentialalgebraic systems), are better represented by a set of differential and algebraic equations describing the generalized dynamic and static behaviors. In the literature, descriptor models have been considered to address a large amount of applications, such as water distribution networks [23], chemical systems [4], electrical circuits [8], [19], aircraft systems [21], biological systems [28] as well as economic systems [27]. For monitoring purposes and for developing control strategies, state estimation is usually required. Some research works on state estimation for discrete-time descriptor systems have been carried out (see as e.g. [10], [11], where system states are estimated by using different versions of Kalman filtering).

\footnotetext{
‡ This work was partially supported by the Spanish State Research Agency (AEI) and the European Regional Development Fund (ERFD) through the projects ECOCIS (ref. DPI2013-48243-C2-1-R), DEOCS (ref. DPI2016-76493-C3-3-R) and HARCRICS (ref. DPI2014-58104-R), and the FPI grant (ref. BES-2014-068319). The material in this paper was not presented at any meeting.

Corresponding author: Y. Wang. Tel. +34-93-4015805. Fax +34-93-4015750.

Email addresses: ywang@iri.upc.edu (Ye Wang), vicenc.puig@upc.edu (Vicenç Puig), cembrano@iri.upc.edu (Gabriela Cembrano).
} 
Research on set-based state estimation has been quite active for the last decades, e.g. [1], [7], [12], [17], [18], [22] among others. In the literature, set-based state estimation approaches can be classified according to whether they follow a set-membership or an interval observer-based paradigm. A set-membership approach relies on over-bounding the uncertain estimated states considering unknown-but-bounded uncertainties [20]. An interval observer-based approach bounds the set of estimated states by means of an observer structure in which the gain is designed assuming that uncertainties are modeled in a deterministic way (as e.g. using intervals for bounding them [9]) or in a stochastic way (as e.g. using the Kalman filtering [13], [14]). From the application point of view, the set-based approaches are very popular in the fault diagnosis framework, e.g. [16], [26], [24].

Zonotopes are a special class of geometrical sets. The symmetry properties of zonotopes help to reduce the computational load of using them in an iterative way. Worst-case state estimation for dynamical systems using zonotopes is investigated in [17]. A state bounding observer based on zonotopes is introduced in [5]. The zonotopic observer in combination with Kalman filtering is addressed in [6], [7]. Moreover, a set-membership approach based on zonotopes is proposed for dynamical systems in [1], [2].

\subsection{Contribution}

The main contribution of this paper is to propose a set-membership state estimator and a zonotopic Kalman observer for discrete-time descriptor systems. Basically, three types of system uncertainties are considered: unknown inputs and unknown-but-bounded system disturbances and measurement noise. One limitation for the use of zonotopic approaches in real applications is that some system disturbances are unknown and it may not be possible to bound them in a predefined zonotope as a-prior knowledge. To overcome this problem, two classes of unknown system disturbances are considered: (i) bounded disturbances in a zonotope; (ii) unbounded disturbances, which are considered to be unknown inputs and can be decoupled in the observer design.

For the proposed set-membership approach, the consistent states with measurements are enclosed by a parameterized intersection zonotope. To reduce the size of the intersection zonotope, the $F_{W}$-radius [6] and $W$-radius [15] criteria are considered. The $F_{W}$-radius criterion is used through the Kalman filtering procedure while the $W$-radius criterion is taken into account via an optimization problem including linear matrix inequalities (LMIs). For the designed zonotopic Kalman observer, we present the explicit solution of the optimal Kalman gain based on the $F_{W}$-radius criterion. Moreover, the relationship between the proposed set-membership approach and zonotopic Kalman observer is discussed.

\subsection{Outline}

The paper is organized as follows. The problem statement is expressed in Section 2. The set-membership approach for discrete-time descriptor systems is proposed in Section 3 and the zonotopic Kalman observer for discrete-time descriptor systems is designed in Section 4. The relationship between both approaches is discussed in Section 5 . A numerical example is provided to illustrate the effectiveness of both approaches and comparison results are also shown in Section 6. Finally, conclusions are presented in Section 7.

Notation. An $m$-order zonotope $\mathcal{Z} \in \mathbb{R}^{n}(m \geq n)$ is defined by a hypercube $\mathbf{B}^{m}=[-1,+1]^{m}$ affine projection with the center $p \in \mathbb{R}^{n}$ and the generator matrix $H \in \mathbb{R}^{n \times m}$ as

$$
\mathcal{Z}=\langle p, H\rangle=\left\{p+H z, z \in \mathbf{B}^{m}\right\}
$$

Denote the Minkowski sum as $\oplus$ and the linear image product as $\odot$. The zonotope $\mathcal{Z}$ in $(1)$ can also be defined by $\mathcal{Z}=p \oplus H \mathbf{B}^{m}$. Besides, the following properties hold:

$$
\begin{aligned}
\left\langle p_{1}, H_{1}\right\rangle \oplus\left\langle p_{2}, H_{2}\right\rangle & =\left\langle p_{1}+p_{2},\left[\begin{array}{ll}
H_{1} & H_{2}
\end{array}\right]\right\rangle \\
L \odot\langle p, H\rangle & =\langle L p, L H\rangle \\
\langle p, H\rangle & \subseteq\langle p, \operatorname{rs}(H)\rangle
\end{aligned}
$$

where $L$ is a matrix of appropriate dimension. $\langle p, r s(H)\rangle$ is called interval hull of the zonotope $\mathcal{Z}=\langle p, H\rangle$ and $r s(H)$ returns a diagonal matrix with diagonal elements of $\operatorname{rs}(H)_{i, i}=\sum_{j=1}^{m}\left|H_{i, j}\right|$ for $i=1, \ldots, n$. 
For $\mathcal{Z}=\langle p, H\rangle$, the weighted zonotope reduction operator proposed in [7] is denoted by $\downarrow_{q, W}(H)$ satisfying the inclusion property $\langle p, H\rangle \subseteq\left\langle p, \downarrow_{q, W}(H)\right\rangle$, where $q \geq n$ specifies the maximum number of columns of $\downarrow_{q, W}(H)$ and $W$ is a weighting matrix of appropriate dimension.

For $X \in \mathbb{R}^{n \times n}$, we use $\operatorname{tr}(X)=\sum_{i=1}^{n} X_{i i}$ and $\operatorname{rank}(X)$ to denote the trace and the rank of $X$, and if $X$ is nonsingular, we use $X^{-1}$ to denote the inverse matrix of $X$. $\operatorname{vec}(X)$ denotes the vectorization of $X . X \succ 0$ denotes positive definiteness if the scalar $x^{T} X x$ is positive for arbitrary non-zero column vector $x$ of real numbers. Similarly, $X \succeq 0$ denotes positive semi-definiteness. If $X$ is symmetric, we use $\star$ to denote a symmetric element in $X$. We use $I_{m}$ to denote an identity matrix of dimension $m$. For two matrices $X$ and $Y$, the Kronecker product of these two matrices is denoted by $X \otimes Y$.

Let $X, A, B$ and $C$ be matrices of appropriate dimensions. The following matrix calculus regarding the matrix trace holds:

$$
\begin{aligned}
\frac{\partial}{\partial X} \operatorname{tr}\left(A X^{T} B\right) & =A^{T} B^{T}, \\
\frac{\partial}{\partial X} \operatorname{tr}\left(A X B X^{T} C\right) & =B X^{T} C A+B^{T} X^{T} A^{T} C^{T} .
\end{aligned}
$$

For $H \in \mathbb{R}^{n \times m}$, with $W \in \mathbb{R}^{n \times n}$ and $W=W^{T} \succ 0$, the weighted Frobenius norm of $H$ is defined by $\|H\|_{F, W}=$ $\sqrt{\operatorname{tr}\left(H^{T} W H\right)}$ and $\|H\|_{F}=\sqrt{\operatorname{tr}\left(H^{T} H\right)}$, obtained with $W=I_{n}$, is the non-weighted Frobenius norm. For $h \in \mathbb{R}^{n}$, the weighted and non-weighted 2-norms of $h$ are denoted by $\|h\|_{2, W}=\sqrt{h^{T} W h}$ and $\|h\|_{2}=\sqrt{h^{T} h}$ obtained with $W=I_{n}$, respectively.

\section{Problem statement}

Consider the discrete-time descriptor linear system as

$$
\begin{aligned}
E x_{k+1} & =A x_{k}+B u_{k}+D \omega_{k}+D_{d} d_{k}, \\
y_{k} & =C x_{k}+F v_{k},
\end{aligned}
$$

where $x \in \mathbb{R}^{n_{x}}$ denotes the vector of system states, $u \in \mathbb{R}^{n_{u}}$ denotes the vector of known inputs, $d \in \mathbb{R}^{n_{d}}$ denotes the vector of unknown inputs, $y \in \mathbb{R}^{n_{y}}$ denotes the vector of measurement outputs, $E \in \mathbb{R}^{n_{x} \times n_{x}}, A \in \mathbb{R}^{n_{x} \times n_{x}}$, $B \in \mathbb{R}^{n_{x} \times n_{u}}, C \in \mathbb{R}^{n_{y} \times n_{x}}, D \in \mathbb{R}^{n_{x} \times n_{w}}, D_{d} \in \mathbb{R}^{n_{x} \times n_{d}}$ and $F \in \mathbb{R}^{n_{y} \times n_{v}}$. Besides, the initial state $x_{0}$ is given in the inclusion zonotope $\mathcal{X}_{0}=\left\langle p_{0}, H_{0}\right\rangle$, where $p_{0} \in \mathbb{R}^{n_{x}}$ and $H_{0} \in \mathbb{R}^{n_{x} \times n_{x}}$ are the center and generator matrix of this zonotope. The system disturbance vector $\omega_{k} \in \mathbb{R}^{n_{w}}$ and measurement noise vector $v_{k} \in \mathbb{R}^{n_{v}}$ are assumed to be unknown but bounded by zonotopes $\omega_{k} \in \mathcal{W}=\left\langle 0, I_{n_{w}}\right\rangle, v_{k} \in \mathcal{V}=\left\langle 0, I_{n_{v}}\right\rangle, \forall k \in \mathbb{N}$.

For the descriptor system (4), $E$ may be a singular matrix and $\operatorname{rank}(E) \leq n_{x}$. Assume that the descriptor system (4) is detectable and the unknown input $d_{k}, \forall k \in \mathbb{N}$ can be decoupled, that is, matrices $E, C$ and $D_{d}$ satisfy the following rank condition:

$$
\operatorname{rank}\left(\left[\begin{array}{c}
I_{n_{x}} \otimes\left[\begin{array}{cc}
E & D_{d} \\
C & 0
\end{array}\right] \\
\operatorname{vec}\left(\left[\begin{array}{c}
I_{n_{x}} \\
0
\end{array}\right]\right)
\end{array}\right]\right)=n_{x} \cdot \operatorname{rank}\left(\left[\begin{array}{cc}
E & D_{d} \\
C & 0
\end{array}\right]\right)
$$

Thus, there exists a nonempty set of solutions of matrices $T$ and $N$ satisfying

$$
\begin{aligned}
T E+N C & =I_{n_{x}}, \\
T D_{d} & =0 .
\end{aligned}
$$


In this paper, we investigate state estimation approaches based on zonotopes for descriptor system (4). We propose two ways to use zonotope bounding uncertain states with unknown but bounded disturbances and noise as well as unbounded disturbances (as unknown inputs). For notation simplicity, the discrete-time instant $k$ is omitted and the time instant $k+1$ is replaced by the subscript + while the subscript - stands for the time instant $k-1$ in the rest of the paper.

\section{Set-membership approach for discrete-time descriptor systems}

In this section, we propose a set-membership state estimation approach based on zonotopes for discrete-time descriptor system (4). This approach uses the structure of the parameterized intersection zonotope for implementing the measurement consistency test including unknown inputs. Some preliminary definitions are introduced as follows.

Definition 1 (Uncertain state set) Given the descriptor system (4) with $x_{0} \in\left\langle p_{0}, H_{0}\right\rangle, \omega \in \mathcal{W}, \forall k \in \mathbb{N}$, the uncertain state set $\overline{\mathcal{X}}$ is defined by

$$
\overline{\mathcal{X}}=\left\{x \in \mathbb{R}^{n_{x}} \mid E x \in A \overline{\mathcal{X}}_{-} \oplus B u_{-} \oplus D_{d} d \oplus D \mathcal{W}\right\} .
$$

Definition 2 (Measurement state set) Given the descriptor system (4), a measurement output vector $y$ and $v \in$ $\mathcal{V}, \forall k \in \mathbb{N}$, the measurement state set $\mathcal{P}$ is defined by $\mathcal{P}=\left\{x \in \mathbb{R}^{n_{x}}|| C x-y \mid \leq F\right\}$.

Definition 3 (Exact uncertain state set) Given the descriptor system (4), a measurement output vector $y$, $\omega \in$ $\mathcal{W}$ and $v \in \mathcal{V}, \forall k \in \mathbb{N}$, the exact uncertain state set $\mathcal{X}$ is defined by $\mathcal{X}=\overline{\mathcal{X}} \cap \mathcal{P}$.

Since $d$ is a unknown input vector, it is not possible to directly characterize the uncertain state set from Definition 1 . Meanwhile, the goal is to approximate the exact uncertain state set $\mathcal{X}$ by an outer approximation of $\mathcal{X}$ for the descriptor system (4) through implementing a measurement consistency test. In general, the proposed set-membership approach includes three steps: (i) prediction step; (ii) measurement step; (iii) correction step.

More specifically, assuming $x \in \mathcal{X} \subseteq \hat{\mathcal{X}}=\langle\hat{p}, \hat{H}\rangle$ at time $k$ that also satisfies $x \in \mathcal{X}_{0}=\left\langle p_{0}, H_{0}\right\rangle$ when $k=$ 0 , these three steps are implemented as follows: (i) compute the predicted uncertain state set $\hat{\mathcal{X}}_{+}$; (ii) compute the measurement state set $\mathcal{P}_{+}$with a measurement output vector $y_{+} ;$(iii) find an intersection zonotope $\hat{\mathcal{X}}_{+}(\Lambda$ ) satisfying $\left\{\hat{\mathcal{X}}_{+} \cap \mathcal{P}_{+}\right\} \subseteq \hat{\mathcal{X}}_{+}(\Lambda)$, where $\Lambda \in \mathbb{R}^{n_{x} \times n_{y}}$ is a correction matrix. The structure of this intersection zonotope is defined as follows.

Theorem 1 (Intersection zonotope for descriptor systems) Given the descriptor system (4), a measurement output vector $y_{+}, x_{0} \in \mathcal{X}_{0}, \omega \in \mathcal{W}, v \in \mathcal{V}, \forall k \in \mathbb{N}, x \in\langle\hat{p}, \hat{H}\rangle \subseteq\langle\hat{p}, \bar{H}\rangle$ with $\bar{H}=\downarrow_{q, W}(\hat{H})$, a correction matrix $\Lambda \in \mathbb{R}^{n_{x} \times n_{y}}, T \in \mathbb{R}^{n_{x} \times n_{x}}$ and $N \in \mathbb{R}^{n_{x} \times n_{y}}$ satisfying (6). Then, $x_{+} \in\left\{\hat{\mathcal{X}}_{+} \cap \mathcal{P}_{+}\right\} \subseteq \hat{\mathcal{X}}_{+}(\Lambda)=\left\langle\hat{p}_{+}(\Lambda), \hat{H}_{+}(\Lambda)\right\rangle$, where

$$
\begin{aligned}
\hat{p}_{+}(\Lambda) & =(I-\Lambda C) T A \hat{p}+(I-\Lambda C) T B u+(N+\Lambda-\Lambda C N) y_{+}, \\
\hat{H}_{+}(\Lambda) & =\left[\begin{array}{llll}
(I-\Lambda C) T A \bar{H} & (I-\Lambda C) T D & (I-\Lambda C) N F & \Lambda F
\end{array}\right]
\end{aligned}
$$

PROOF. For any $x_{+} \in\left\{\hat{\mathcal{X}}_{+} \cap \mathcal{P}_{+}\right\}$, we have $x_{+} \in \hat{\mathcal{X}}_{+}$and $x_{+} \in \mathcal{P}_{+}$. For the descriptor system (4a) with the inclusion $x \in\langle\hat{p}, \hat{H}\rangle \subseteq\left\langle\hat{p}, \downarrow_{q, W}(\hat{H})\right\rangle$, there exists a vector $s_{1} \in \mathbf{B}^{q+n_{w}}$ such that $E x_{+}=A \hat{p}+B u+D_{d} d+[A \bar{H} D] s_{1}$. Besides, from $x_{+} \in \mathcal{P}_{+}$, there exists a vector $\alpha \in \mathbf{B}^{n_{v}}$ such that

$$
C x_{+}-y_{+}=F \alpha
$$

Consider the rank condition (5) is satisfied. With a pair of matrices $T$ and $N$ satisfying (6), (4) and (8) can be combined leading to

$$
(T E+N C) x_{+}=T A \hat{p}+T B u+T D_{d} d+N y_{+}+[T A \bar{H} T D] s_{1}+N F \alpha .
$$


Set $R=[T A \bar{H} T D N F]$ and $\beta=\left[\begin{array}{ll}s_{1}^{T} & \alpha^{T}\end{array}\right]^{T}$. According to (6), the above equation can be simplified to be

$$
x_{+}=T A \hat{p}+T B u+N y_{+}+R \beta .
$$

Therefore, with $\Lambda \in \mathbb{R}^{n_{x} \times n_{y}}$ and a correction term $\Lambda C R \beta$, we add and substitute $C R \beta$ in (9) to obtain

$$
x_{+}=T A \hat{p}+T B u+N y_{+}+\Lambda C R \beta+(I-\Lambda C) R \beta .
$$

By substituting $x_{+}$in (8) by (9), we also have $C R \beta=y_{+}-C N y_{+}-C T A \hat{p}-C T B u+F \alpha$. And then by replacing $C R \beta$ in (10), we have

$$
\begin{aligned}
x_{+}= & (I-\Lambda C) T A \hat{p}+(I-\Lambda C) T B u \\
& +(N+\Lambda-\Lambda C N) y_{+}+[(I-\Lambda C) R \Lambda F]\left[\begin{array}{l}
\beta \\
\alpha
\end{array}\right] .
\end{aligned}
$$

Thus, we obtain $\hat{p}_{+}(\Lambda)$ and $\hat{H}_{+}(\Lambda)$ as in $(7)$.

Due to the intersection zonotope bounding uncertain states including propagated estimation errors and uncertainties, we would like to find a suitable correction matrix minimizing the effects of estimation errors and uncertainties by reducing the size of the intersection zonotope. To measure the size of a zonotope, the $F_{W}$-radius and the $W$-radius are considered as follows.

Definition 4 ( $F_{W}$-radius) Given a weighting matrix $W \in \mathbb{R}^{n_{x} \times n_{x}}, W=W^{T} \succ 0$, the $F_{W}$-radius of the zonotope $\mathcal{Z}=\langle p, H\rangle$ is defined using the weighted Frobenius norm of $H$ as $\ell_{F, W}=\|\langle p, H\rangle\|_{F, W}=\|H\|_{F, W}$.

Definition 5 (W-radius) Given a weighting matrix $W \in \mathbb{R}^{n_{x} \times n_{x}}, W=W^{T} \succ 0$, the $W$-radius of the zonotope $\mathcal{Z}=$ $\langle p, H\rangle$ with $H \in \mathbb{R}^{n_{x} \times r}$ is defined as $\ell_{W}=\max _{z \in \mathcal{Z}}\|z-p\|_{2, W}^{2}=\max _{b \in \mathbf{B}^{r}}\|H b\|_{2, W}^{2}$.

In the following, we first compute a time-varying Kalman correction matrix based on the $F_{W}$-radius. On the other hand, with a $W$-radius minimization criterion, a correction matrix can be obtained by solving an off-line optimization problem. This off-line correction matrix can also be updated following an on-line updating procedure.

\subsection{Compute the correction matrix via Kalman filtering procedure}

From Definition 4 , the size of the intersection zonotope $\hat{\mathcal{X}}_{+}(\Lambda)$ can be measured by the $F_{W}$-radius as

$$
\begin{aligned}
\ell_{F, W_{+}} & =\left\|\hat{H}_{+}(\Lambda)\right\|_{F, W}^{2}=\operatorname{tr}\left(\hat{H}_{+}^{T}(\Lambda) W \hat{H}_{+}(\Lambda)\right) \\
& =\operatorname{tr}\left(W \hat{H}_{+}(\Lambda) \hat{H}_{+}^{T}(\Lambda)\right)=\operatorname{tr}\left(W P_{+}(\Lambda)\right),
\end{aligned}
$$

where $P_{+}(\Lambda)=\hat{H}_{+}(\Lambda) \hat{H}_{+}^{T}(\Lambda)$. As in the Kalman filtering procedure described in [7, Theorem 5], a Kalman correction matrix $\Lambda^{*}$ can be obtained by minimizing $\ell_{F, W_{+}}$of the intersection zonotope $\left\langle\hat{p}_{+}(\Lambda), \hat{H}_{+}(\Lambda)\right\rangle$.

Theorem 2 (Kalman correction matrix) Given the intersection zonotope $\hat{\mathcal{X}}_{+}(\Lambda)=\left\langle\hat{p}_{+}(\Lambda), \hat{H}_{+}(\Lambda)\right\rangle$ in $(7)$ and a weighting matrix $W=W^{T} \succ 0$. The optimal correction matrix $\Lambda^{*}$ minimizes $J=\ell_{F, W_{+}}$and its explicit solution 
is given by

$$
\begin{aligned}
\Lambda^{*} & =L S^{-1}, \\
L & =\bar{R} C^{T}, \\
S & =C \bar{R} C^{T}+Q_{v}, \\
\bar{R} & =T\left(A \bar{P} A^{T}+Q_{w}\right) T^{T}+N Q_{v} N^{T},
\end{aligned}
$$

with $\bar{P}=\bar{H} \bar{H}^{T}, Q_{w}=D D^{T}$ and $Q_{v}=F F^{T}$.

PROOF. From (7), we have

$$
\begin{aligned}
P_{+}(\Lambda)= & (I-\Lambda C) T A \bar{P} A^{T} T^{T}(I-\Lambda C)^{T} \\
& +(I-\Lambda C) T Q_{w} T^{T}(I-\Lambda C)^{T} \\
& +(I-\Lambda C) N Q_{v} N^{T}(I-\Lambda C)^{T}+\Lambda Q_{v} \Lambda^{T}
\end{aligned}
$$

The criterion $J=\ell_{F, W_{+}}$is being convex with respect to $\Lambda$. By setting $L, S$ and $\bar{R}$ as in (13), (14) and (15), we take the partial-derivative of $J=\ell_{F, W_{+}}$in (11) with respect to $\Lambda$ to obtain $\frac{\partial}{\partial \Lambda} \operatorname{tr}\left(W P_{+}(\Lambda)\right)=\frac{\partial}{\partial \Lambda} \operatorname{tr}\left(W \Lambda S \Lambda^{T}\right)-$ $2 \frac{\partial}{\partial \Lambda} \operatorname{tr}\left(W L \Lambda^{T}\right)$. Therefore, $\Lambda^{*}$ is the value of $\Lambda$ such that $\frac{\partial}{\partial \Lambda} \operatorname{tr}\left(W P_{+}(\Lambda)\right)=0$. By using (3a) and (3b), we have $S \Lambda^{* T} W+S^{T} \Lambda^{* T} W^{T}-2 L^{T} W^{T}=0$. Since that $S$ is also symmetric, we then obtain $W \Lambda^{*} S=W L$, which leads to $(12)$.

From Theorem 2, the optimal correction matrix $\Lambda^{*}$ is independent of the weighting matrix $W$. Hence, $W$ can be set as free and we can also use the non-weighted Frobenius norm to measure the zonotope size as the $F$-radius.

\subsection{Compute the correction matrix using optimization-based methods}

From Definition 5 , the size of the intersection zonotope $\hat{\mathcal{X}}_{+}(\Lambda)$ can also be measured by the $W$-radius as

$$
\begin{aligned}
\ell_{W_{+}} & =\max _{z \in \mathbf{B}^{\left(q+n_{x}+2 n_{y}\right)}}\left\|\hat{H}_{+}(\Lambda) z\right\|_{2, W}^{2} \\
& =z^{T} \hat{H}_{+}^{T}(\Lambda) W \hat{H}_{+}(\Lambda) z .
\end{aligned}
$$

A $W$-radius minimization criterion and the corresponding LMI condition are presented in the following theorem.

Theorem 3 (W-radius minimization criterion) Given the intersection zonotope $\hat{\mathcal{X}}_{+}(\Lambda)=\left\langle\hat{p}_{+}(\Lambda), \hat{H}_{+}(\Lambda)\right\rangle$ in $(7), \gamma \in(0,1)$ and $\epsilon>0$. The zonotope minimization criterion

$$
\ell_{W_{+}} \leq \gamma \ell_{W}+\epsilon
$$

holds if there exist matrices $W \in \mathbb{R}^{n_{x}}, W=W^{T} \succ 0, Y \in \mathbb{R}^{n_{x} \times n_{y}}$, diagonal matrices $\Gamma \in \mathbb{R}^{n_{x} \times n_{x}}, \Upsilon \in \mathbb{R}^{n_{y} \times n_{y}}$, $\Omega \in \mathbb{R}^{n_{y} \times n_{y}}$ such that

$$
\left[\begin{array}{ccccc}
\gamma W & \star & \star & \star & \star \\
0 & \Gamma & \star & \star & \star \\
0 & 0 & \Upsilon & \star & \star \\
0 & 0 & 0 & \Omega & \star \\
(W-Y C) T A & (W-Y C) T D & (W-Y C) N F & Y F & W
\end{array}\right] \succ 0,
$$




$$
\begin{aligned}
& \Gamma \succ 0, \Upsilon \succ 0, \Omega \succ 0, \\
& \operatorname{tr}(\Gamma)+\operatorname{tr}(\Upsilon)+\operatorname{tr}(\Omega)<\epsilon .
\end{aligned}
$$

PROOF. By combining (16) and (17), we have

$$
\max _{z \in \mathbf{B}^{\left(q+n_{x}+2 n_{y}\right)}}\left\|\hat{H}_{+}(\Lambda) z\right\|_{2, W}^{2}-\max _{\bar{z} \in \mathbf{B}^{q}} \gamma\|\bar{H} \bar{z}\|_{2, W}^{2}-\epsilon \leq 0 .
$$

For any $\bar{H}$ and $\bar{z} \in \mathbf{B}^{q}, \max _{\bar{z} \in \mathbf{B}^{q}}\|\bar{H} \bar{z}\|_{2, W}^{2} \geq\|\bar{H} \bar{z}\|_{2, W}^{2}$. Set $z=\left[\begin{array}{llll}\bar{z}^{T} & b_{1}^{T} & b_{2}^{T} & b_{3}^{T}\end{array}\right]^{T} \in \mathbf{B}^{\left(q+n_{w}+2 n_{v}\right)}$ with $\bar{z} \in \mathbf{B}^{q}, b_{1} \in \mathbf{B}^{n_{w}}$, $b_{2} \in \mathbf{B}^{n_{v}}$ and $b_{3} \in \mathbf{B}^{n_{v}}$. Then, we obtain a sufficient condition for any $z \in \mathbf{B}^{\left(q+n_{w}+2 n_{v}\right)}$ and $\bar{z} \in \mathbf{B}^{q}$

$$
\left\|\hat{H}_{+}(\Lambda) z\right\|_{2, W}^{2}-\gamma\|\bar{H} \bar{z}\|_{2, W}^{2}-\epsilon<0
$$

Recall $\hat{H}_{+}(\Lambda)$ in $(7 \mathrm{~b})$ and set $Y=W \Lambda$. Let us denote

$$
\tilde{R}=\left[\begin{array}{lll}
(W-Y C) T A & (W-Y C) T D & (W-Y C) N F \quad Y F
\end{array}\right]
$$

Therefore, (21) can be reformulated as

$$
\left[\begin{array}{c}
\bar{H} \bar{z} \\
b_{1} \\
b_{2} \\
b_{3}
\end{array}\right]^{T} \tilde{R}^{T} W^{-1} \tilde{R}\left[\begin{array}{c}
\bar{H} \bar{z} \\
b_{1} \\
b_{2} \\
b_{3}
\end{array}\right]-\gamma \bar{z}^{T} \bar{H}^{T} W \bar{H} \bar{z}-\epsilon<0
$$

for any $\bar{z} \in \mathbf{B}^{q}, b_{1} \in \mathbf{B}^{n_{w}}, b_{2} \in \mathbf{B}^{n_{v}}$ and $b_{3} \in \mathbf{B}^{n_{v}}$. If $\Gamma, \Upsilon$ and $\Omega$ are diagonal positive semi-definite matrices, then we have $b_{1}^{T} \Gamma b_{1}=\sum_{i=1}^{n_{x}} b_{1}^{2} \Gamma_{i} \leq \operatorname{tr}(\Gamma), b_{2}^{T} \Upsilon b_{2}=\sum_{i=1}^{n_{y}} b_{2}^{2} \Upsilon_{i} \leq \operatorname{tr}(\Upsilon), b_{3}^{T} \Omega b_{3}=\sum_{i=1}^{n_{y}} b_{3}^{2} \Omega_{i} \leq \operatorname{tr}(\Omega)$, for any $b_{1} \in \mathbf{B}^{n_{x}}$, $b_{2} \in \mathbf{B}^{n_{y}}$ and $b_{3} \in \mathbf{B}^{n_{y}}$, where $\Gamma_{i}, \Upsilon_{i}$ and $\Omega_{i}$ are each diagonal element of $\Gamma, \Upsilon$ and $\Omega$. Therefore, we obtain

$$
\begin{aligned}
& \operatorname{tr}(\Gamma)-b_{1}^{T} \Gamma b_{1} \geq 0, \forall b_{1} \in \mathbf{B}^{n_{x}} \\
& \operatorname{tr}(\Upsilon)-b_{2}^{T} \Upsilon b_{2} \geq 0, \forall b_{2} \in \mathbf{B}^{n_{y}} \\
& \operatorname{tr}(\Omega)-b_{3}^{T} \Omega b_{3} \geq 0, \forall b_{3} \in \mathbf{B}^{n_{y}}
\end{aligned}
$$

By adding (24) to (23), we obtain a sufficient condition of (23)

$$
\begin{aligned}
& {\left[\begin{array}{c}
\bar{H} \bar{z} \\
b_{1} \\
b_{2} \\
b_{3}
\end{array}\right] \quad \tilde{R}^{T} W^{-1} \tilde{R}\left[\begin{array}{c}
\bar{H} \bar{z} \\
b_{1} \\
b_{2} \\
b_{3}
\end{array}\right]-\gamma \bar{z}^{T} \bar{H}^{T} W \bar{H} \bar{z}} \\
& \quad+\operatorname{tr}(\Gamma)-b_{1}^{T} \Gamma b_{1}+\operatorname{tr}(\Upsilon)-b_{2}^{T} \Upsilon b_{2} \\
& \quad+\operatorname{tr}(\Omega)-b_{3}^{T} \Omega b_{3}-\epsilon<0 .
\end{aligned}
$$


If (20) holds, then we obtain

$$
\left[\begin{array}{c}
\bar{H} \bar{z} \\
b_{1} \\
b_{2} \\
b_{3}
\end{array}\right]^{T}\left(\tilde{R}^{T} W^{-1} \tilde{R}-\left[\begin{array}{cccc}
\gamma W & 0 & 0 & 0 \\
0 & \Gamma & 0 & 0 \\
0 & 0 & \Upsilon & 0 \\
0 & 0 & 0 & \Omega
\end{array}\right]\right)\left[\begin{array}{c}
\bar{H} \bar{z} \\
b_{1} \\
b_{2} \\
b_{3}
\end{array}\right]<0
$$

Again, from the above inequality, we have a sufficient condition

$$
\left[\begin{array}{cccc}
\gamma W & 0 & 0 & 0 \\
0 & \Gamma & 0 & 0 \\
0 & 0 & \Upsilon & 0 \\
0 & 0 & 0 & \Omega
\end{array}\right]-\tilde{R}^{T} W^{-1} \tilde{R} \succ 0
$$

By using the Schur complement and $\tilde{R}$ in (22), we obtain (18).

Proposition 1 (Ultimate bound of the $W$-radius) Given the intersection zonotope $\hat{\mathcal{X}}_{+}(\Lambda)=\left\langle\hat{p}_{+}(\Lambda), \hat{H}_{+}(\Lambda)\right\rangle$ in $(7), \gamma \in(0,1)$ and $\epsilon>0$. If the criterion (17) holds, then the $W$-radius of intersection zonotope $\hat{\mathcal{X}}_{+}(\Lambda)$ is ultimately bounded by

$$
\ell_{W, \infty} \leq \frac{\epsilon}{1-\gamma}
$$

PROOF. With given $\gamma \in(0,1)$ and $\epsilon>0$, we take $k \rightarrow \infty$ in (17) to obtain $\ell_{W, \infty} \leq \gamma \ell_{W, \infty}+\epsilon$ that implies the ultimate bound $(25)$ of $\ell_{W}$.

Since (25) characterizes an ellipsoid with given $\gamma \in(0,1)$ and $\epsilon>0$, in order to minimize the ultimate bound $\ell_{W, \infty}$, we can maximize a norm of $W$. For instance, we choose to maximize $\operatorname{tr}(W)$. Therefore, the optimization problem to find the off-line correction matrix $\Lambda_{f}$ can be expressed as

$$
\min _{W, Y, \Gamma, \Upsilon, \Omega}-\operatorname{tr}(W)
$$

subject to (18)-(20). The optimal solution of the optimization problem (26) gives $\Lambda_{f}=W^{-1} Y$.

To tighten the size of the intersection zonotope during iterations, we also introduce an on-line method to update the correction matrix $\Lambda_{o}$ with the weighting matrix $W$ obtained by solving (26).

Theorem 4 Given the intersection zonotope $\hat{\mathcal{X}}_{+}(\Lambda)=\left\langle\hat{p}_{+}(\Lambda), \hat{H}_{+}(\Lambda)\right\rangle$ in $(7)$ and the matrix W obtained by solving (26). If there exists a diagonal matrix $M \in \mathbb{R}^{n_{x} \times n_{x}}$ such that

$$
\left[\begin{array}{cc}
M & \star \\
W \hat{H}_{+}(\Lambda) & W
\end{array}\right] \succ 0
$$

then $\ell_{W_{+}}$in $(16)$ is bounded by

$$
\ell_{W_{+}}<\max _{z \in \mathbf{B}^{\left(q+n_{x}+2 n_{y}\right)}}\|M z\|_{2}^{2}
$$


PROOF. According to [3], the vertices of the intersection zonotope $\hat{\mathcal{X}}_{+}(\Lambda)$ can be approximated by using a diagonal matrix. With a diagonal matrix $M \in \mathbb{R}^{n_{x} \times n_{x}}$, a sufficient condition of (28) can be obtained as

$$
z^{T} \hat{H}_{+}^{T}(\Lambda) W \hat{H}_{+}(\Lambda) z<z^{T} M z, \forall z \in \mathbf{B}^{\left(q+n_{x}+2 n_{y}\right)}
$$

Then, from above, we have a sufficient condition $M-\hat{H}_{+}^{T}(\Lambda) W \hat{H}_{+}(\Lambda) \succ 0$. By using the Schur complement, we obtain (27).

At each time step, minimizing the size of the intersection zonotope measured by the $W$-radius $\ell_{W}$ can be implemented by minimizing the trace of the diagonal matrix $M$. Therefore, the on-line updating correction matrix $\Lambda_{o}$ can be obtained by solving the following optimization problem:

$$
\min _{\Lambda} \operatorname{tr}(M)
$$

subject to $(27)$.

Remark 1 It is worth mentioning that the off-line correction matrix $\Lambda_{f}$ could be already useful for estimating the states. Hence, sometimes $\Lambda_{o}$ obtained through the on-line updating implementation with (29) does not provide significant improvements since the state estimations are already satisfactory in terms of degrees of freedom of the intersection zonotope defined in (7).

\section{Zonotopic Kalman observer for discrete-time descriptor systems}

In this section, we design a zonotopic Kalman observer for the descriptor system (4). Unlike the set-membership approach proposed in Section 3, this zonotopic observer structure is defined based on the Luenberger observer structure.

\subsection{Zonotopic observer structure for descriptor systems}

With $T$ and $N$ satisfying (6), we consider the Luenberger observer structure for the descriptor system (4) in a prediction type [25] as

$$
\begin{aligned}
\hat{x}_{+}= & T A \hat{x}+T B u+T D \omega+T D_{d} d+N y_{+} \\
& -N F v_{+}+G(y-C \hat{x}-F v)
\end{aligned}
$$

where $\hat{x} \in \mathbb{R}^{n_{x}}$ denotes the estimated state vector, $G \in \mathbb{R}^{n_{x} \times n_{y}}$ denotes the time-varying observer gain.

For the descriptor system (4), we would like to bound the uncertain system states $x, \forall k \in \mathbb{N}_{+}$in a zonotopic set. A suitable observer gain $G$ is used to reduce the state estimation error with a measurement output $y$. We first recursively define the structure of the zonotopic observer.

Theorem 5 (Prediction-type zonotopic observer for descriptor systems) Given the descriptor system in (4), measurement output vectors $y, y_{+}, x_{0} \in \mathcal{X}_{0}, \omega \in \mathcal{W}, v \in \mathcal{V}, \forall k \in \mathbb{N}, x \in\langle\hat{p}, \hat{H}\rangle \subseteq\langle\hat{p}, \bar{H}\rangle$ with $\bar{H}=\downarrow_{q}, W \quad(\hat{H})$, $T \in \mathbb{R}^{n_{x} \times n_{x}}$ and $N \in \mathbb{R}^{n_{x} \times n_{y}}$ satisfying (6). The zonotope bounding uncertain states can be recursively defined by $x_{+} \in \hat{\mathcal{X}}_{+}(G)=\left\langle\hat{p}_{+}(G), \hat{H}_{+}(G)\right\rangle$, where

$$
\begin{aligned}
\hat{p}_{+}(G) & =(T A-G C) \hat{p}+T B u+G y+N y_{+}, \\
\hat{H}_{+}(G) & =[(T A-G C) \bar{H} T D-N F-G F] .
\end{aligned}
$$


PROOF. Assuming that $x \in\langle\hat{p}, \bar{H}\rangle$, we set $\hat{x}=x \in\langle\hat{p}, \bar{H}\rangle$. Since $\omega \in \mathcal{W}, v \in \mathcal{V}$, from (30), we have

$$
\begin{aligned}
x_{+} \in & \hat{\mathcal{X}}_{+}(G)=\left\langle\hat{p}_{+}(G), \hat{H}_{+}(G)\right\rangle \\
= & ((T A-G C) \odot\langle\hat{p}, \bar{H}\rangle) \oplus(T B \odot\langle u, 0\rangle) \\
& \oplus(G \odot\langle y, 0\rangle) \oplus\left(N \odot\left\langle y_{+}, 0\right\rangle\right) \oplus\left(T D \odot\left\langle 0, I_{n_{w}}\right\rangle\right) \\
& \oplus\left((-N F) \odot\left\langle 0, I_{n_{v}}\right\rangle\right) \oplus\left((-G F) \odot\left\langle 0, I_{n_{v}}\right\rangle\right) .
\end{aligned}
$$

By using properties in (2) to the above equation, we obtain $\hat{p}_{+}(G)$ and $\hat{H}_{+}(G)$ as in (31).

From the state bounding zonotope in (31), the state estimation error $\varepsilon_{+}$is bounded by the zonotope $\varepsilon_{+}=x_{+}-$ $\hat{p}_{+}(G) \in \mathcal{E}_{x}=\left\langle 0, \hat{H}_{+}(G)\right\rangle$. The objective for the zonotopic observer design is to find a time-varying observer gain $G$ to minimize the estimation error, that corresponds to the size of $\mathcal{E}_{x}$.

\subsection{Optimal Kalman observer gain for descriptor systems}

As in Theorem 2, the minimization criterion is based on the $F_{W}$-radius. The optimal observer gain $G^{*}$ can be found by minimizing the $F_{W}$-radius of $\mathcal{E}_{x}, \tilde{J}=\operatorname{tr}\left(W \tilde{P}_{+}(G)\right)$ with $\tilde{P}_{+}(G)=\hat{H}_{+}(G) \hat{H}_{+}^{T}(G)$.

Theorem 6 (Optimal Kalman observer gain for descriptor systems) Given $\mathcal{E}_{x}=\left\langle 0, \hat{H}_{+}(G)\right\rangle$ with $\hat{H}_{+}(G)$ in (31b) and $W=W^{T} \succ 0$. The optimal observer gain $G^{*}$ minimizes $\tilde{J}=\operatorname{tr}\left(W \tilde{P}_{+}(G)\right)$ and its explicit solution is given by

$$
\begin{aligned}
G^{*} & =T A K, \\
K & =\tilde{L} \tilde{S}^{-1}, \\
\tilde{L} & =\tilde{P} C^{T}, \\
\tilde{S} & =C \tilde{P} C^{T}+Q_{v},
\end{aligned}
$$

with $\tilde{P}=\bar{H} \bar{H}^{T}$ and $Q_{v}=F F^{T}$.

PROOF. From (31b), we have $\tilde{P}_{+}(G)=(T A-G C) \bar{H} \bar{H}^{T}(T A-G C)^{T}+T D D^{T} T^{T}+N F F^{T} N^{T}+G F F^{T} G^{T}$. Since $\tilde{J}$ is convex with respect to $G, G^{*}$ is the value of $G$ such that $\frac{\partial}{\partial G} \operatorname{tr}\left(W \tilde{P}_{+}(G)\right)=0$. By setting $\tilde{L}$ and $\tilde{S}$ as $(34)$ and (35), we have $\frac{\partial}{\partial G} \operatorname{tr}\left(W G \tilde{S} G^{T}\right)-2 \frac{\partial}{\partial G} \operatorname{tr}\left(W T A \tilde{L} G^{T}\right)=0$.

Due to the symmetry of $\tilde{S}$, by using (3a) and (3b), we obtain $W G^{*} \tilde{S}=W T A \tilde{L}$. Set $K$ as in (33). Thus, $G^{*}$ can be found in (32).

From Theorem $6, G^{*}$ is also independent of the weighting matrix $W$. To make use of $\downarrow_{q, W}(\cdot)$, a weighting matrix $W$ is required. One selection of $W$ is proposed in the following proposition.

Proposition 2 Given the nominal descriptor system $E x_{+}=A x+B u$ and $y=C x$, matrices $T \in \mathbb{R}^{n_{x} \times n_{x}}$ and $N \in$ $\mathbb{R}^{n_{x} \times n_{y}}$ satisfying (6a). The Luenberger observer defined by $\hat{x}_{+}=T A \hat{x}+T B u+\bar{G}(y-C \hat{x})+N y_{+}$is $\mu$-stable (stable with a decay rate $\mu$ ) if there exists $W \in \mathbb{R}^{n_{x}}, W=W^{T} \succ 0, Y \in \mathbb{R}^{n_{x} \times n_{y}}$, and a scalar $\mu \in(0,1]$ such that

$$
\left[\begin{array}{cr}
\mu W & \star \\
W T A-Y C & W
\end{array}\right] \succ 0 .
$$


PROOF. With matrices $T$ and $N$ satisfying (6a), the nominal system dynamics can be expressed as $x_{+}=T A x+$ $T B u+N y_{+}$. Let us define the state estimation error $e=x-\hat{x}$. Therefore, we have the error dynamics $e_{+}=$ $x_{+}-\hat{x}_{+}=(T A-\bar{G} C) e$.

With $W=W^{T} \succ 0$, the Lyapunov candidate function is chosen as $V=e^{T} W e$. Then, with $\mu \in(0,1]$, we have $\Delta V=e_{+}^{T} W e_{+}-e^{T} \mu W e=e^{T}(T A-\bar{G} C)^{T} W(T A-\bar{G} C) e-e^{T} \mu W e$. For $e \neq 0, \Delta V<0$ gives $\mu W-$ $(T A-\bar{G} C)^{T} W(T A-\bar{G} C) \succ 0$. By applying the Schur complement lemma with $\mu W \succ 0$ and $Y=W \bar{G}$, we obtain (36).

For the nominal descriptor system $E x_{+}=A x+B u$, the observer gain without taking into account system uncertainties can also be found by satisfying (36) with $\bar{G}=W^{-1} Y$. We will use $\bar{G}$ with the zonotopic observer structure defined in (31) to compare with $G^{*}$ in order to assess the state bounding performance.

\section{On the relationship between set-membership approach and zonotopic observer}

\subsection{Relationship between two proposed approaches}

Comparing the parameterized intersection zonotope structure proposed in Theorem 1 and the zonotopic observer structure proposed in Theorem 5 , the intersection zonotope is formulated by considering the measurement output $y_{+}$ to implement the system consistency test while the zonotopic observer includes measurement outputs $y$ and $y_{+}$.

To find the relationship between these two approaches, we also consider a current estimation-type zonotopic observer for the descriptor system (4) only containing the current measurement output $y_{+}$as follows:

$$
\begin{aligned}
\hat{x}_{+}= & T A \hat{x}+T B u+T D_{d} d+T D \omega+N y_{+} \\
& -N F v_{+}+\hat{G}\left(y_{+}-C \check{x}_{+}-F v_{+}\right),
\end{aligned}
$$

where $\hat{G} \in \mathbb{R}^{n_{x} \times n_{y}}$ is a time-varying observer gain for the current estimation-type zonotopic observer. $\check{x}_{+}$denotes the predicted state from the previous observed state $\hat{x}$ that can be computed by

$$
\check{x}_{+}=T A \hat{x}+T B u+T D \omega+N y_{+}-N F v_{+} .
$$

Theorem 7 Consider the descriptor system (4). The proposed set-membership approach is equivalent to the current estimation-type zonotopic observer in the structure of (37).

PROOF. In terms of the zonotopic observer in the current estimation-type, by substituting $\check{x}_{+}$by (38) to (37), we have

$$
\begin{aligned}
\hat{x}_{+}= & (I-\hat{G} C) T A \hat{x}+(I-\hat{G} C) T B u \\
& +(I-\hat{G} C) T D \omega+(N+\hat{G}-\hat{G} C N) y_{+} \\
& -(I-\hat{G} C) N F v_{+}-\hat{G} F v_{+} .
\end{aligned}
$$

Considering $x \in\langle\hat{p}, \bar{H}\rangle$ with $\bar{H}=\downarrow_{q, W}(\hat{H}), \omega \in \mathcal{W}$ and $v_{+} \in \mathcal{V}$, the uncertain state $x_{+}$is bounded into the 
zonotope $\tilde{\mathcal{X}}_{+}(\hat{G})=\left\langle\tilde{p}_{+}(\hat{G}), \tilde{H}_{+}(\hat{G})\right\rangle$, where

$$
\begin{aligned}
x_{+} \in & \tilde{\mathcal{X}}_{+}(\hat{G})=\left\langle\tilde{p}_{+}(\hat{G}), \tilde{H}_{+}(\hat{G})\right\rangle \\
= & ((I-\hat{G} C) T A \odot\langle p, \bar{H}\rangle) \oplus((I-\hat{G} C) T B \odot\langle u, 0\rangle) \\
& \oplus\left((I-\hat{G} C) T D \odot\left\langle 0, I_{n_{w}}\right\rangle\right) \\
& \oplus\left((N+\hat{G}-\hat{G} C N) \odot\left\langle y_{+}, 0\right\rangle\right) \\
& \oplus\left((-(I-\hat{G} C)) N F \odot\left\langle 0, I_{n_{v}}\right\rangle\right) \\
& \oplus\left((-\hat{G} F) \odot\left\langle 0, I_{n_{v}}\right\rangle\right) .
\end{aligned}
$$

By using properties in $(2)$, we obtain $\tilde{p}_{+}(\hat{G})$ and $\tilde{H}_{+}(\hat{G})$ as follows:

$$
\begin{aligned}
\tilde{p}_{+}(\hat{G}) & =(I-\hat{G} C) T A p+(I-\hat{G} C) T B u+(N+\hat{G}-\hat{G} C N) y_{+}, \\
\tilde{H}_{+}(\hat{G}) & =\left[\begin{array}{llll}
(I-\hat{G} C) T A \bar{H} & (I-\hat{G} C) T D & -(I-\hat{G} C)) N F & -\hat{G} F
\end{array}\right] .
\end{aligned}
$$

By definition of the zonotope, the subtraction sign in the last two terms of (39b) can be removed. Therefore, (7) and (39) are equivalent with $\Lambda=\hat{G}$.

Remark 2 Since the structure of $\left\langle\tilde{p}_{+}(\hat{G}), \tilde{H}_{+}(\hat{G})\right\rangle$ is equivalent to the intersection zonotope $\left\langle\hat{p}_{+}(\Lambda), \hat{H}_{+}(\Lambda)\right\rangle$ in $(7)$, the observer gain $\hat{G}$ can be obtained by using methods proposed for the set-membership approach in Section 3.

\subsection{Extension to dynamical systems with unknown inputs}

In the case of $\operatorname{rank}(E)=n_{x}$, the system (4) becomes a dynamical system. The unknown input $d$ can be decoupled by finding matrices $\bar{T} \in \mathbb{R}^{n_{x} \times n_{x}}$ and $\bar{N} \in \mathbb{R}^{n_{x} \times n_{y}}$ that satisfy

$$
\begin{aligned}
\bar{T}+\bar{N} C & =I_{n_{x}}, \\
\bar{T} D_{d} & =0 .
\end{aligned}
$$

By combining (40a) and (40b), we obtain $D_{d}=\bar{N} C D_{d}$ and $\bar{T}=I_{n_{x}}-\bar{N} C$. Assume $D_{d}$ to be full column rank. The condition to guarantee the existence of $\bar{T}$ and $\bar{N}$ is given by $\operatorname{rank}\left(D_{d}\right)=\operatorname{rank}\left(C D_{d}\right)$. In this case, the proposed set-membership approach and zonotopic Kalman observer in Section 3 and 4 can be applied to dynamical systems subject to unknown inputs, which can be considered an improvement on the methods presented in [1], [7]. Under this structure with $\bar{T}$ and $N$, the effects of unknown inputs can be decoupled. We reduce the limitation of zonotope-based approach, that is, the system disturbances are not required to be bounded.

\section{Illustrative example}

To illustrate the proposed state estimation approaches, a discrete-time descriptor system as defined in (4) is considered with

$$
E=\left[\begin{array}{lll}
1 & 0 & 0 \\
0 & 1 & 0 \\
0 & 0 & 0
\end{array}\right], A=\left[\begin{array}{ccc}
0.5 & 0 & 0 \\
0.8 & 0.95 & 0 \\
-1 & 0.5 & 1
\end{array}\right], B=\left[\begin{array}{ll}
1 & 0 \\
0 & 1 \\
0 & 0
\end{array}\right], D_{d}=\left[\begin{array}{c}
0 \\
0 \\
0.8
\end{array}\right]
$$




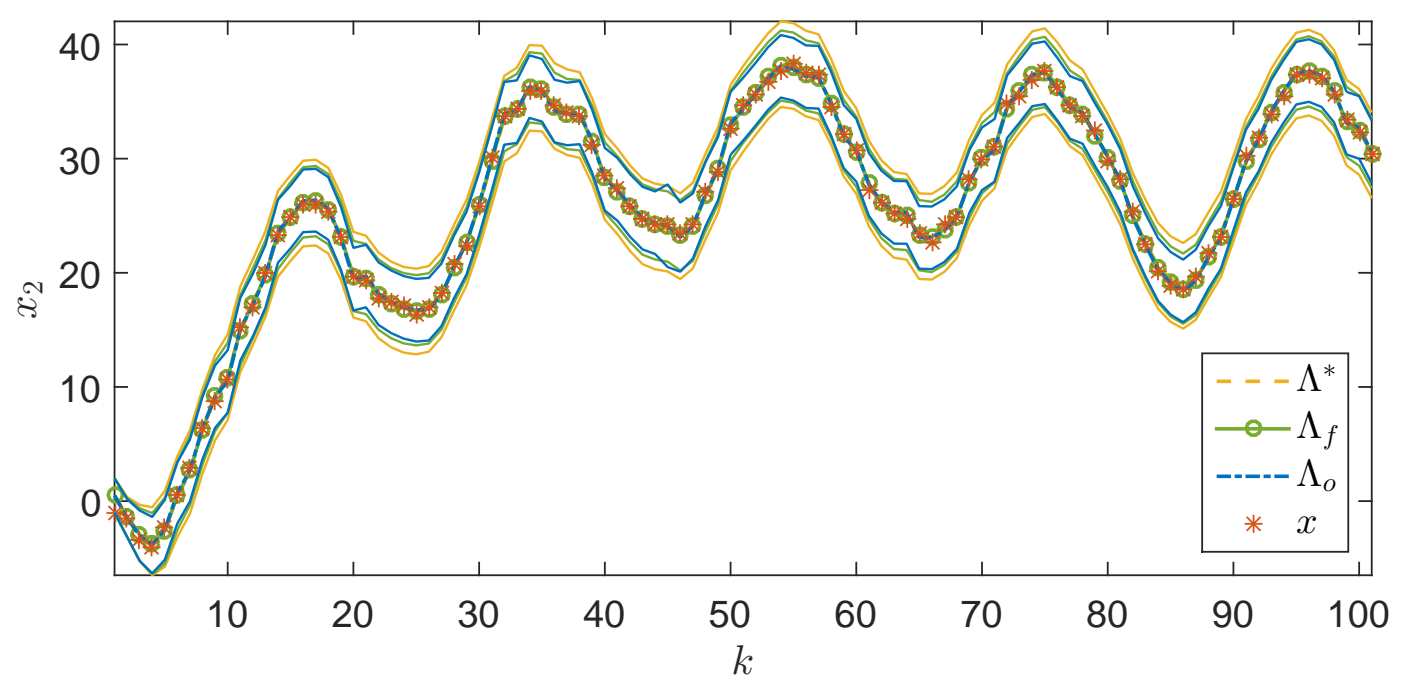

Fig. 1. The result by applying the set-membership approach.

$$
C=\left[\begin{array}{ccc}
1 & 0 & 1 \\
1 & -1 & 0
\end{array}\right], D=\left[\begin{array}{ccc}
0.1 & 0 & 0 \\
0 & 1.5 & 0 \\
0 & 0 & 0.6
\end{array}\right], F=\left[\begin{array}{cc}
0.5 & 0 \\
0 & 1.5
\end{array}\right] \text {. }
$$

The input signal $u$ is given by $u=\left[\begin{array}{c}0.5 \sin (t)+1 \\ -2 \cos (t)\end{array}\right]$, for $t \in[0,10 \pi]$ with 100 sampling steps. The system disturbances $\omega$ and measurement noise $v$ are random Gaussian white noise bounded in zonotopes: $\omega \in \mathcal{W}=\left\langle 0, I_{3}\right\rangle$ and $v \in\left\langle 0, I_{2}\right\rangle$, $\forall k \in \mathbb{N}$. Since $E, C$ and $D_{d}$ satisfy the rank condition (5), there exists a solution of matrices $T$ and $N$ satisfying (6). Therefore, we choose one solution, for instance

$$
T=\left[\begin{array}{ccc}
0.6667 & 0.3333 & 0 \\
0.3333 & 0.6667 & 0 \\
-0.6667 & -0.3333 & 0
\end{array}\right], N=\left[\begin{array}{cc}
0 & 0.3333 \\
0 & -0.3333 \\
1 & -0.3333
\end{array}\right] .
$$

The initial state zonotope $\mathcal{X}_{0}$ is given by $\mathcal{X}_{0}=\left\langle p_{0}, H_{0}\right\rangle$, with $p_{0}=\left[\begin{array}{lll}0.5 & 0.5 & 0.25\end{array}\right]^{T}$ and $H_{0}=\left[\begin{array}{ccc}0.1 & 0 & 0 \\ 0 & 1.5 & 0 \\ 0 & 0 & 0.6\end{array}\right]$. The real uncertain initial state vector is selected as $x_{0}=\left[\begin{array}{lll}0.5 & 0.5 & 0.25\end{array}\right]^{T}$. We choose $q=15$ in the zonotope reduction operator to reduce the computation load and simulation time. Simulations have been carried out in a PC with the CPU of Intel (R) Core (TM) i7-5500U 2.4GHz, 12GB RAM and MATLAB R2015a. As a result, the state estimation results are shown in Fig. 1 and 2. These plots show that both the set-membership approach and the zonotopic Kalman observer are able to provide the interval-based state estimation results.

Recall $\Lambda^{*}$ as Kalman correction matrix, $\Lambda_{f}$ obtained by solving the off-line optimization problem (26), $\Lambda_{o}$ obtained by solving the on-line optimization problem (29), $G^{*}$ as the optimal Kalman gain and $\bar{G}$ with $\mu=1$ as the nominal observer gain of the prediction-type zonotopic Kalman observer, and $\hat{G}$ as the optimal Kalman gain of the current estimation type. Besides, the optimal weighting matrix $W^{*}$ is obtained also by solving (26). The observation error is defined as $e_{:}=x_{:}-\hat{x}_{:}=x_{:}-p_{:}$, where $\hat{x}_{:} \in\left\langle p_{:}, H_{:}\right\rangle$and the subscript : represents any time instant $k \in \mathbb{N}$. The mean square error (MSE) between the real uncertain states and observed states can be computed by $M S E\left(e_{:}\right)=\left(\frac{1}{N} \sum_{k=1}^{N} \frac{1}{n_{x}}\left\|e_{:}\right\|_{2}^{2}\right)^{\frac{1}{2}}$. Besides, we also compute the root mean squared value of $r s\left(H_{:}\right)$that is denoted by $\operatorname{RMS}\left(r s\left(H_{:}\right)\right)$. The weighted and non-weighted Frobenius norm, the weighted 2-norm of the segment 


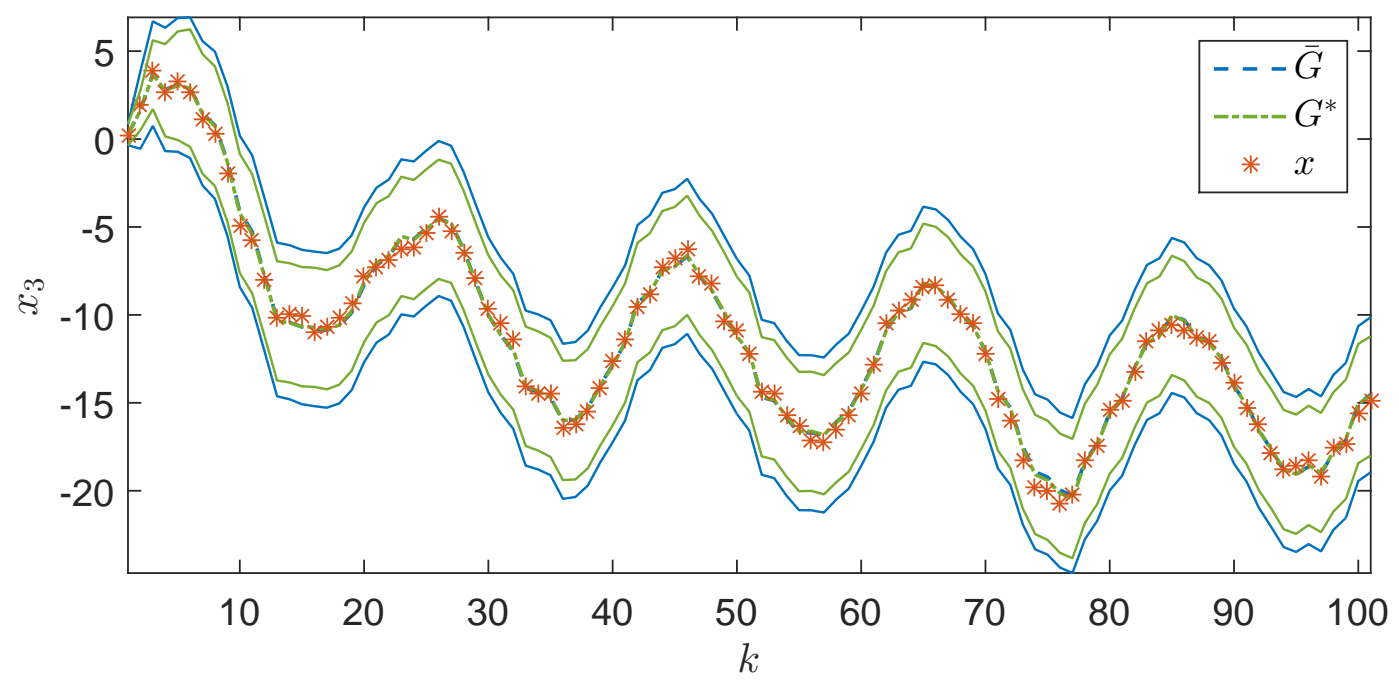

Fig. 2. The result by applying the prediction-type zonotopic Kalman observer.

Table 1

Comparison results with weighted zonotope reduction operator $\downarrow_{q, W}(H)$

\begin{tabular}{cccccccc} 
Approach & $\Lambda / G$ & $M S E\left(e_{:}\right)$ & $\operatorname{RMS}\left(\operatorname{rs}\left(H_{:}\right)\right)$ & $\left\|H_{:}\right\|_{F}$ & $\left\|H_{:}\right\|_{F, W}$ & $\left\|H_{:}\right\|_{2, W}$ & Time $[\mathrm{s}]$ \\
\hline \multirow{2}{*}{ Set-membership approach } & $\Lambda^{*}$ & 0.0355 & 3.0963 & 1.5485 & 0.7070 & - & - \\
& $\Lambda_{f}$ & 0.0326 & 2.5443 & - & - & 14.1385 & 3.809 \\
& $\Lambda_{o}$ & 0.0287 & 2.4755 & - & - & 14.1373 & 46.238 \\
\hline \multirow{2}{*}{ Zonotopic observer } & $G^{*}$ & 0.2528 & 3.8781 & 1.9694 & 1.9594 & - & - \\
& $\bar{G}$ & 0.3027 & 5.0544 & 2.2605 & 2.2490 & - & - \\
\hline
\end{tabular}

Table 2

Comparison results with non-weighted zonotope reduction operator $\downarrow_{q}(H)$

\begin{tabular}{cccccccc}
\hline Approach & $\Lambda / G$ & $M S E\left(e_{:}\right)$ & $\operatorname{RMS}\left(r s\left(H_{:}\right)\right)$ & $\left\|H_{:}\right\|_{F}$ & $\left\|H_{:}\right\|_{F, W}$ & $\left\|H_{:}\right\|_{2, W}$ & Time $[\mathrm{s}]$ \\
\hline \multirow{3}{*}{ Set-membership approach } & $\Lambda^{*}$ & 0.0539 & 3.1970 & 1.5110 & 0.7070 & - & - \\
& $\Lambda_{f}$ & 0.0404 & 2.5542 & - & - & 14.1385 & 2.909 \\
& $\Lambda_{o}$ & 0.0365 & 2.5466 & - & - & 14.1373 & 44.158 \\
\hline \multirow{2}{*}{ Zonotopic observer } & $G^{*}$ & 0.2118 & 3.9386 & 1.9737 & 1.2672 & - & - \\
& $\bar{G}$ & 0.2335 & 5.0927 & 2.2357 & 1.9308 & - & - \\
\hline
\end{tabular}

matrix of zonotopes are computed to compare the sizes of the state zonotopes for all the scenarios. Table 1 and 2 show comparison results of all the cases in the root mean square up to the step 100 with weighted and non-weighted zonotope reduction operator.

From the $\operatorname{MSE}\left(e_{\mathrm{:}}\right)$ results of $\Lambda^{*}$ and $G^{*}$ in Table 1, the performance of the set-membership approach is better than the zonotopic Kalman observer in prediction-type. This is because the prediction-type observer structure includes two consecutive-step measurement outputs and noise. Both the measurement noise $v$ and $v_{+}$should be over-approximated by the terms $-N F$ and $-G F$ in (31b). Hence, this could enlarge the size of the zonotope and gives more conservative estimation intervals. In terms of the real-time implementation of control loops, in both proposed approaches, the estimate $x_{+}$depends on $y_{+}$. Hence, a state feedback control like $u_{+}=K x_{+}$cannot be applied at the same time 
as $y_{+}$is acquired. However, this real-time synchronization difficulty does not exist when implementing a control loop based on the zonotopic Kalman filter in prediction-type form for dynamical systems as proposed in [7]. Hence, a real-time synchronization remains an open problem when implementing a state feedback control loop with the proposed state estimators for descriptor systems.

From the results with $\Lambda_{f}$ and $\Lambda_{o}$, the mean-square error and the size of the intersection zonotopes using the on-line method are smaller than the one using the off-line method. According to $\operatorname{RMS}\left(\operatorname{rs}\left(H_{:}\right)\right)$and $\left\|H_{\text {: }}\right\|_{2, W}$ of the setmembership approach, the on-line method improves the correction matrix $\Lambda$ with the weighting matrix $W$ computed off line. Since the optimization problem (29) is implemented on line, the simulation time is longer than the off-line method. For the prediction-type zonotopic Kalman observer, the optimal Kalman gain $G^{*}$ deals with uncertainties better than the nominal observer gain $\bar{G}$.

Besides, by comparing the first and last rows of Table 1 and 2, it is numerically shown that the set-membership approach is equivalent to the current estimation-type zonotopic Kalman observer as the discussion in Theorem 7 . From Table 2, all the approaches are run with non-weighted zonotope reduction operator $\downarrow_{q}(H)$. From results of $\operatorname{RMS}\left(r s\left(H_{:}\right)\right)$, the size of each zonotope is larger than the case with $\downarrow_{q, W}(H)$. This is because the non-weighted zonotope reduction operator can bring more over-approximated results.

\section{Conclusion}

In this paper, we have proposed a set-membership state estimation approach and a zonotopic Kalman observer for discrete-time descriptor systems subject to uncertainties and unknown inputs. In the proposed set-membership approach, we provide several methods for finding the correction matrix to characterize the intersection zonotope. In the proposed zonotopic Kalman observer, we propose the optimal Kalman observer gain in prediction estimation-type. Furthermore, we prove that the zonotopic Kalman observer in the current estimation-type structure is equivalent to the proposed set-membership approach. As a future research, we will extend the results presented in this paper to non-linear systems using as e.g. an LPV representation.

\section{Acknowledgements}

We would like to thank Prof. Teodoro Alamo for useful discussions about this work. We also acknowledge editors and anonymous reviewers for useful suggestions to help us improve this paper.

\section{References}

[1] T. Alamo, J.M. Bravo, and E.F. Camacho. Guaranteed state estimation by zonotopes. Automatica, 41(6):1035-1043, 2005.

[2] T. Alamo, J.M. Bravo, M.J. Redondo, and E.F. Camacho. A set-membership state estimation algorithm based on DC programming. Automatica, 44(1):216-224, 2008.

[3] T. Alamo, R. Tempo, D.R. Ramírez, and E.F. Camacho. A new vertex result for robustness problems with interval matrix uncertainty. Systems \& Control Letters, 57(6):474-481, 2008.

[4] L. Biegler, S. Campbell, and V. Mehrmann. Control and Optimization with Differential-Algebraic Constraints. Society for Industrial and Applied Mathematics, Philadelphia, USA, 2012.

[5] C. Combastel. A state bounding observer based on zonotopes. In European Control Conference (ECC), pages 2589-2594, 2003.

[6] C. Combastel. Merging Kalman filtering and zonotopic state bounding for robust fault detection under noisy environment. In 9 th IFAC Symposium on Fault Detection, Supervision and Safety for Technical Processes (SAFEPROCESS), volume 48, pages 289-295, 2015.

[7] C. Combastel. Zonotopes and Kalman observers: Gain optimality under distinct uncertainty paradigms and robust convergence. Automatica, 55:265-273, 2015.

[8] G.R. Duan. Analysis and Design of Descriptor Linear Systems. Springer, New York, USA, 2010.

[9] D. Efimov, W. Perruquetti, T. Raïssi, and A. Zolghadri. Interval observers for time-varying discrete-time systems. IEEE Transactions on Automatic Control, 58(12):3218-3224, 2013.

[10] C.S. Hsieh. State estimation for descriptor systems via the unknown input filtering method. Automatica, 49(5):1281-1286, 2013.

[11] J.Y. Ishihara, M.H. Terra, and A.F. Bianco. Recursive linear estimation for general discrete-time descriptor systems. Automatica, 46(4):761-766, 2010.

[12] L. Jaulin, M. Kieffer, O. Didrit, and E. Walter. Applied Interval Analysis, with Examples in Parameter and State Estimation, Robust Control and Robotics. Springer, 2001. 
[13] R.E. Kalman. A new approach to linear filtering and prediction problems. ASME Journal of Basic Engineering”, 82(1):35-45, 1960.

[14] R.E. Kalman and R.S. Bucy. New results in linear filtering and prediction theory. ASME Journal of Basic Engineering", 83(1):95$108,1961$.

[15] V.T.H. Le, C. Stoica, T. Alamo, E.F. Camacho, and D. Dumur. Zonotopic guaranteed state estimation for uncertain systems. Automatica, 49(11):3418-3424, 2013.

[16] V. Puig. Fault diagnosis and fault tolerant control using set-membership approaches: Application to real case studies. International Journal of Applied Mathematics and Computer Science, 20(4):619-635, 2010.

[17] V. Puig, P. Cuguero, and J. Quevedo. Worst-case state estimation and simulation of uncertain discrete-time systems using zonotopes. In European Control Conference, pages 1691-1697, 2001.

[18] T. Raïssi, N. Ramdani, and Y. Candau. Set membership state and parameter estimation for systems described by nonlinear differential equations. Automatica, 40(10):1771-1777, 2004.

[19] R. Riaza. Differential-algebraic systems: Analytical aspects and circuit applications. World Scientific Publishing Company, New York, USA, 2008.

[20] F. Schweppe. Recursive state estimation: Unknown but bounded errors and system inputs. IEEE Transactions on Automatic Control, 13(1):22-28, 1968.

[21] B.L. Stevens, F. L. Lewis, and E.N. Johnson. Aircraft Control and Simulation: Dynamics, Controls Design, and Autonomous Systems. Wiley-Blackwell, New York, USA, 2016.

[22] R.E.H. Thabet, T. Raïssi, C. Combastel, D. Efimov, and A. Zolghadri. An effective method to interval observer design for timevarying systems. Automatica, 50(10):2677-2684, 2014.

[23] Y. Wang, V. Puig, and G. Cembrano. Non-linear economic model predictive control of water distribution networks. Journal of Process Control, 56:23-34, 2017.

[24] Y. Wang, Z. Wang, V. Puig, and G. Cembrano. Zonotopic fault estimation filter design for discrete-time descriptor systems. In 20th IFAC World Congress, pages 5211-5216, Toulouse, France, 2017.

[25] Z. Wang, Y. Shen, X. Zhang, and Q. Wang. Observer design for discrete-time descriptor systems: An LMI approach. Systems \& Control Letters, 61(6):683-687, 2012.

[26] F. Xu, V. Puig, C. Ocampo-Martinez, F. Stoican, and S. Olaru. Actuator-fault detection and isolation based on set-theoretic approaches. Journal of Process Control, 24(6):947-956, 2014.

[27] L. Zhang, J. Lam, and Q. Zhang. Lyapunov and Riccati equations of discrete-time descriptor systems. IEEE Transactions on Automatic Control, 44(11):2134-2139, 1999.

[28] Q.L. Zhang, C. Liu, and X. Zhang. Complexity, Analysis and Control of Singular Biological Systems. Springer, London, UK, 2012. 


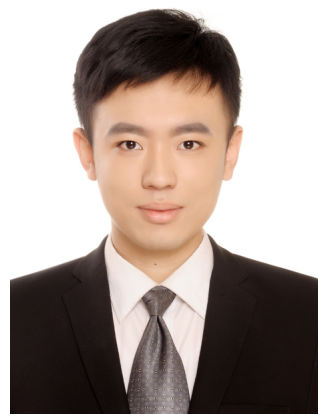

Ye Wang received his B.Sc. degree in Engineering from the Northeast Forestry University, Harbin, P.R. China in 2012, and M.Sc. degree in Automatic Control and Robotics from the Technical University of Catalonia (UPC), Barcelona, Spain, in 2014. Currently, he is a Ph.D. candidate at the UPC and a research fellow of Spanish National Research Council (CSIC) at the Institute of Robotics and Industrial Informatics (IRI), CSIC-UPC. He has participated in the European project EFFINET (ref. FP7-ICT-2011-8-31855) and the Spanish projects ECOCIS (ref. DPI2013-48243-C2-1-R), HARCRICS (ref. DPI201458104-R) and DEOCS (ref. DPI2016-76493-C3-3-R). His current research interests include economic model predictive control, set-theoretic fault diagnosis and fault-tolerant control with application to water systems and smart grids.

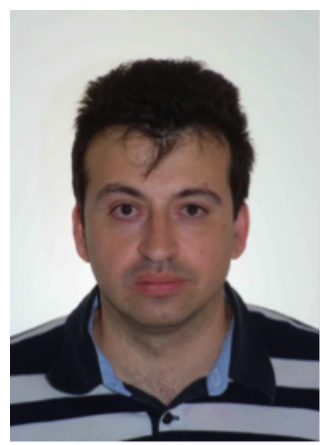

Vicenç Puig received his B.Sc./M.Sc. Degree in Telecommunications Engineering in 1993 and Ph.D. degree in Automatic Control, Vision and Robotics in 1999, both from the Technical University of Catalonia (UPC). He is a full professor at the Automatic Control Department of the UPC and a researcher at the Institute of Robotics and Industrial Informatics (IRI), CSIC-UPC. He is the chair of the Automatic Control Department and the head of the research group on Advanced Control Systems (SAC) at the UPC. He has developed important scientific contributions in the areas of fault diagnosis and fault tolerant control, using interval and linear-parameter-varying models using set-based approaches. He has participated in more than 20 European and national research projects in the last decade. He has also led many private contracts with several companies and has published more than 140 journal articles as well as over 400 contributions in international conference/workshop proceedings. He has supervised over $20 \mathrm{PhD}$ dissertations and over 50 Master's theses/final projects. He is currently the vice-chair of the IFAC Safeprocess TC Committee 6.4 (2014-2018). He was the general chair of the 3rd IEEE Conference on Control and Fault-Tolerant Systems (SysTol 2016) and is the IPC chair of IFAC Safeprocess 2018.

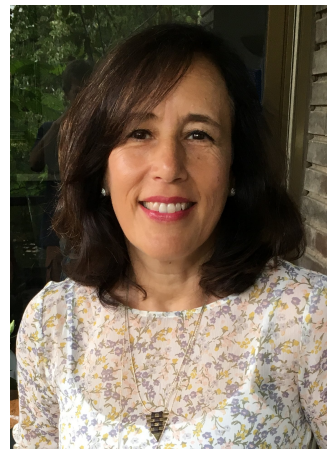

Gabriela Cembrano received her M.Sc. and Ph.D. degrees, in Industrial Engineering and Automatic Control respectively, from the Technical University of Catalonia-BarcelonaTech. She is tenured researcher of the Spanish National Research Council (CSIC) at the Institute of Robotics and Industrial Computing (IRI). Since 2007, she is also a Scientific Advisor of CETaqua Water technology Centre. Her main research area is Control Engineering and its applications to water systems management. She has taken part in numerous fundamental and industrial research projects in this field since 1990. Most recently, she has been Scientific Director of EC project EFFINET Integrated Real-time Monitoring and Control of Drinking Water Networks and she is currently CSIC leader in project LIFE-EFFIDRAIN Efficient Integrated Real-time Control in Urban Drainage and Wastewater Treatment plants for Environmental Protection and in National Research Grant DPI2016-76493-C3-3-R Data-driven Monitoring, Diagnosis and Fault-tolerant Control of Cyber-physical Systems. She has published over 50 journal and conference papers in the field. 